\title{
Unruhe in der Mitte: Die geprellten Leistungsträger des Aufschwungs
}

\author{
Michael Vester \\ Christel Teiwes-Kügler
}

\begin{abstract}
Die Furcht vor der Globalisierung hat die soziale Mitte erreicht. Zu den Schattenseiten des Booms gehören nicht allein die klassischen Mittel- und Arbeitslosen am Rande des Existenzminimums. Auch für diejenigen, die beschäftigt und integriert sind, werden soziale Standards unsicher, verschwinden qualifizierte Arbeitsplätze ins Ausland und ist die Hälfte der neuen Arbeitsplätze prekär. In einer explorativen Pilotstudie sind wir den Fragen nachgegangen, warum auch gut qualifizierte moderne Leistungsträger sich ihrer Lage nicht mehr sicher sind, wie diese Unsicherheit mit zunehmenden innerbetrieblichen Interessenkonflikten verbunden ist und warum trotzdem die gewerkschaftlichen Interessenvertretungen nur bedingt neue Mitglieder finden. ${ }^{1}$
\end{abstract}

\section{Akzeptanzkrise der Gewerkschaften?}

Untersucht wurden diese Phänomene am Beispiel der größten und exportstärksten Branchen, in der Metall- und Elektroindustrie. Seit den 1990er Jahren verlieren die großen Industriegewerkschaften vor allem dort an Mitgliedern, wo eine rasche Modernisierung einen tief greifenden Strukturwandel der Beschäftigten nach sich zieht. $^{2}$ Der Anteil un- und angelernter Arbeitskräfte, die gut organisierte Stammklientel der IG Metall, nimmt immer mehr ab; der der Angestellten, zu denen immer mehr qualifizierte Fachkräfte mit akademischen Berufsabschlüssen gehören, ist auf mehr als die Hälfte der Beschäftigten gewachsen. ${ }^{3}$ Doch ihre Mitgliedschaft in der IG Metall stagniert bei etwa $17 \%$. In den schrumpfenden Bereichen der taylorisierten Massenarbeit sind mehr als $40 \%$, in den wachsenden moderneren Facharbeitermilieus um die $26 \%$ gewerkschaftlich organisiert.

Diese Zahlen liegen im internationalen Vergleich zwar in der Mitte, und es gibt inzwischen auch positive Gegentrends. Aber warum nehmen die Mitgliederzahlen in modernen Branchen meist noch ab, dort wo die Arbeit qualifizierter und eigenverantwortlicher wird? Hat hier eine „Individualisierungstendenz" den Gegensatz zwischen Kapital und Arbeit abgeschwächt? Oder hat dieser Gegensatz nur eine neue Form angenommen? Was trägt hierzu die doppelte Umstrukturierung bei, die $\mathrm{Zu}$ nahme der höher Qualifizierten und die gleichzeitige Verunsicherung durch globale Konkurrenz? Sind schließlich die Ge- werkschaften in ihrem Kommunikationsstil und ihren Tarifstrategien noch zu sehr der alten, gering qualifizierten Arbeiterschaft verhaftet, die ihre Identität in körperlicher Arbeit und kollektiver Kampfkraft suchte?

\section{Der neue industrielle Konflikt: Berufsethos versus Kapitalverwertung}

Die Unübersichtlichkeit der Lage entsteht, weil widersprüchliche Tendenzen zusammenkommen. Die innerbetrieblichen Beziehungen sind durch zwei widerstreitende Prozesse bestimmt, die beide aus der im Weltmaßstab sich verändernden Arbeitsteilung entspringen:

- Berufsethos: Auf der Seite der Arbeitskräfte beschleunigt die internationale Konkurrenz die langfristige Erhöhung der Arbeitsproduktivität durch ständig steigende Bildungsniveaus und Arbeitsqualifikationen, durch ein „re-skilling“. Der Umfang der höher qualifizierten Berufsgruppen, die geringer qualifizierte Gruppen hinter sich lassen, nimmt erheblich zu. Dies fördert gleichzeitig die Renaissance eines $B e$ rufsethos der guten Fach- und Expertenarbeit, bei der es um die Autonomie der Arbeitenden und um einen hohen Gebrauchswert der Arbeitsprodukte geht.

- Kapitalverwertung: Auf der Seite der Unternehmen beschleunigt die internationale Konkurrenz die Tendenz, die Profitabilität des Kapitals durch verschiedene Strategien der Entwertung der Arbeitskraft zu stabilisieren oder zu steigern. Damit verschärfen sich auch die Auseinandersetzungen zwischen verschiedenen Fraktionen der Beschäftigten.

Auf der Leitungsebene verschärfen sich Konflikte zwischen betriebswirtschaftlichen und technischen, kurzfristig und

1 Die Pilotstudie "Neue Arbeitnehmer" wurde 2005 am Institut für Politische Wissenschaft der Universität Hannover durchgeführt. Organisiert wurde sie in Zusammenarbeit mit dem Vorstand der Industriegewerkschaft Metall in Frankfurt/M., der die große Organisation mit ihren vielfältigen Berufsgruppen stärker auf die modernen Arbeitnehmermilieus orientieren will. Die Projektleitung lag bei Michael Vester und Andrea Lange-Vester, die Bearbeitung bei Christel Teiwes-Kügler, unterstützt von Sylvia Heise. Ein ausführlicher Forschungsbericht erscheint demnächst.

2 Die Verluste reichen von ca. $4 \%$ im Fahrzeugbau, $12 \%$ im Maschinenbau bis zu $32 \%$ in der Elektroindustrie (IG Metall 2004).

3 In Nordwürttemberg-Nordbaden ist der Anteil der Angestellten seit 1970 von $26 \%$ auf $39,7 \%$ gestiegen, im Maschinenbau sogar auf $50 \%$. Der Anteil der qualifizierten Facharbeiter ist von $20,5 \%$ auf $26,4 \%$ der Gesamtbeschäftigten gestiegen, der Anteil einfacher, unqualifizierter Arbeiter von $19,3 \%$ auf $6 \%$ zurückgegangen ( $\mathrm{Hu}$ ber 2003).

Michael Vester, Dr. phil., Prof. i. R., Universität Hannover. Arbeitsschwerpunkte: Politische Soziologie und Politische Sozialstrukturanalyse. e-mail:m.vester@agis.uni-hannover.de Christel Teiwes-Kügler, Dipl.-Sozialwiss., Institut für Politische Wissenschaft der Universität Hannover. Arbeitsschwerpunkte: Habitus- und Milieuanalyse, Soziale Ungleichheit und Bildung, Qualitative Methoden der Sozialforschung. e-mail: teiwes-kuegler@t-online.de 
langfristig denkenden Managementfraktionen. Kurzfristige, neoliberale Gewinnstrategien zielen darauf, die Arbeitskraft durch Kostendruck, Flexibilisierung und Arbeitszerlegung, ein neues „de-skilling“, $\mathrm{zu}$ entwerten. Langfristig angelegte, konservative Gewinnstrategien sind darauf ausgerichtet, die steigende Arbeitsproduktivität über paternalistische Strukturen der Betriebsfamilie (aus)zunutzen, wie etwa im Toyota-Modell (Schumann et al. 2006).

Die Gewerkschaften reagieren mit verschiedenen Strategien: Defensive Strategien suchen die Entwertung der Arbeitskraft und die künstliche Vermehrung eines gering qualifizierten „Prekariats“ einzudämmen. Neue offensive Strategien der Gegenmachtpolitik wollen auch das in Bewegung geratene Gefüge der qualifizierten und hoch qualifizierten Berufsgruppen zum Verhandlungs- und Konfliktgegenstand machen, etwa Modelle eines betriebsnahen Produktivitäts- und Mitbestimmungspaktes, wie es das Entgeltrahmenabkommen (ERA) und seit 2004 das Pforzheimer Abkommen möglich machen. ${ }^{4}$ Sie wollen die zunehmende Qualifizierung der Beschäftigten fördern und die dadurch erzielten Produktivitätsgewinne und Standortsicherungen zwischen Unternehmen und Belegschaften tarifvertraglich teilen und über Mitbestimmungsformen regeln (Schumann et al. 2006).

\section{Die Renaissance des Arbeitsethos}

Um ihre Mitgliederpotenziale zu erschließen, müssen sich die Gewerkschaften allerdings auf einen neuen Arbeitnehmertypus einstellen, auf immer höher qualifizierte Spezialisten, denen es - anders als den taylorisierten Massenarbeitern und mehr noch als den spezialisierten Facharbeitern nicht allein um das Geld, sondern auch um die Autoritäts- und Anerkennungsverhältnisse im Betrieb geht. Die Gewerkschaften können immer weniger allein von den ökonomischen Interessen an höheren Löhnen, geringeren Arbeitszeiten und sozialer Sicherheit ausgehen. Im Vordergrund stehen diese eher für „entfremdete“ Routinearbeiter, die vor allem um des Entgelts willen arbeiten müssen. Demgegenüber ist im Zuge des zunehmenden "re-skilling " bis in die industrielle Fertigung hinein eine Wieder- belebung der Facharbeit und ihres „Berufsethos" zu beobachten (u.a. Blauner 1964; Mallet 1972; Piore/Sabel 1985; Kern/Schumann 1984; Grusky/Soerensen 2001). Die Arbeitskräfte haben höhere Ansprüche an Selbstverwirklichung, Fachkönnen und Gestaltungsspielräume. Gleichzeitig erfordert die komplexere Arbeitsorganisation Problemlösungsstrategien und soziale Kompetenzen wie Überblicks- und Kooperationsfähigkeit. Dem entspricht ein eigener Habitus, bei dem statt (männlicher) Körper- und Kampfkraft auch reflexives Argumentationsvermögen, rationale Verständigungsformen und persönliche Autonomie wichtig werden.

Diese Entwicklung wird in der Forschung aufgenommen durch die These einer zunehmenden, obgleich nicht umfassenden, Renaissance berufsspezifischer Fachkulturen (Grusky/Soerensen 2001; Durkheim 1988). Danach gewinnen verbindende moralische Regeln der Zusammenarbeit, der Berufsehre, der Ansprüche an die Arbeitsweise und an die Produktqualität, die mit milieuspezifischen Alltagskulturen, Bildungs- und Gesellungspraktiken verbunden sind, wieder mehr an Gewicht. Beispielsweise ist im Berufsethos der Ingenieure die individuelle und kreative Problemlösung ein zentrales Motiv, aber sie ist eingebunden in den Zusammenhang und die Normen der Berufsgruppe. Sie ist die primäre Referenzgruppe, noch vor dem Unternehmen, von dem als Gegenleistung ein besonderes Maß an Honorierung und Vertrauen erwartet wird. Berufsethos und Berufsgruppe sind der Angelpunkt der Identität, an dem kein anderer Akteur, weder Management noch Gewerkschaft, vorbeikommt.

\section{Der Statusverlust der Leistungsträger}

Diese relative Privilegierung, im Verein mit dem Anspruch an "gute fachliche Arbeit" und "gute Arbeitsprodukte", hat für hoch Qualifizierte lange auch zur Identifikation mit Unternehmenszielen beigetragen (Baethge et al. 1995; Kotthoff 1998; Faust et al.2000). Dieser ständisch privilegierte Status wird heute durch die beiden erwähnten Entwicklungstendenzen untergraben.

Zum einen lässt der funktionale Bedarf den Umfang der immer besser qualifizier- ten Armee von technischen Experten erheblich wachsen. Dadurch verlieren die höheren Berufsqualifikationen den Seltenheitswert, der sie früher vor Entlassungen oder Herabstufungen schützte. Höhere Qualifikation als solche privilegiert nicht mehr - entsprechend dem Satz: „Wenn alle sich auf die Zehenspitzen stellen, sieht keiner besser."

Zum anderen setzen eben daran unternehmerische Strategien an, die das Gros der Ingenieure und technischen Experten als austauschbare Kräfte mit weniger Rechten, Einkommen und Statussicherheit behandeln - wie die übrigen qualifizierten Arbeitnehmer auch. Der Druck zu mehr Flexibilität, Kosten- und Zeitkürzungen ist nur die Spitze des Eisbergs. Hinzu kommen Strategien der Herabstufung, die bis in die Berufsausbildung zurückverlängert werden. In diese werden neue halb- und vorakademische Etagen eingezogen, sodass die anspruchsvolle Arbeit der akademischen Ingenieure (mit Diplom und Master) auch von Protoakademikern (Bachelor, Fachhochschulabsolventen), von Halbakademikern (Fachschulabsolventen) oder Nichtakademikern ausgeführt werden kann. Die Auslagerung der Arbeit an hoch qualifizierte, aber wohlfeilere Experten der neuen Industrieländer ist nur die Fortsetzung dieser Politik der Unsicherheit, durch die das Interesse an einer kollektiven Interessenvertretung, die den sozialen Status der verschiedenen Arbeitnehmergruppen sichert, wieder wachsen könnte.

Die neue Hierarchisierung und Verfügbarmachung umfasst schließlich auch eine Politik der Lebensphasen. Die verbesserten Berufsausbildungen ziehen nach sich, dass immer weniger junge Arbeitnehmer und Arbeitnehmerinnen (wie früher) mit etwa 15 Jahren von der Hauptschule in den Be-

\footnotetext{
4 Mit dem Tarifvertrag des IG Metall Bezirks BadenWürttemberg von Februar 2004 in Pforzheim wurden erstmals betriebsinterne Lösungen und $\mathrm{Ab}$ weichungen vom Tarifvertrag zugelassen, die in Umstellungskrisen der Standort- und Arbeitsplatzsicherung dienen sollen. Der Tarifvertrag wurde innerhalb der IG Metall kontrovers diskutiert und als Zäsur wahrgenommen. Betriebliche Öffnungsklauseln wurden z. B. vorgesehen für (1) eine differenziertere, am betrieblichen Qualifizierungsniveau orientierte Tarifpolitik, (2) flexiblere betriebliche Arbeitszeitmodelle, einschließlich Arbeitszeitverlängerungen auf bis zu 40 Stunden je Woche und (3) Kürzungen von Sonderzahlungen/Ver schiebungen von Sonderansprüchen.
} 


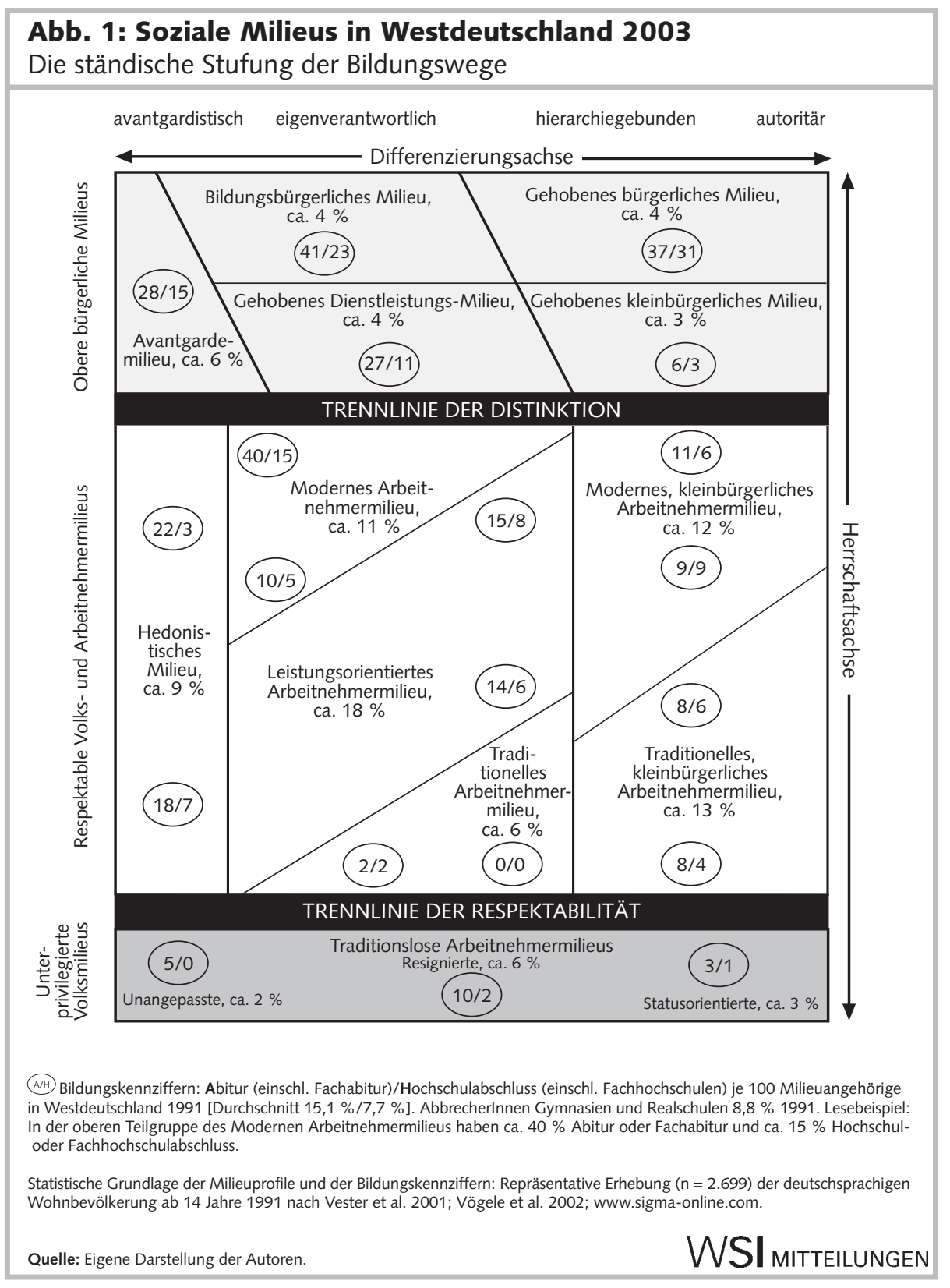

trieb wechseln, wo sie durch die älteren Kolleginnen und Kollegen eine „erste gewerkschaftliche Sozialisation" erhalten. Durch die erheblich verlängerten Ausbildungszeiten bleiben sie auch länger unabhängiger und offener orientiert. Unsere Untersuchung sollte daher auch einbeziehen, wie weit sich die Einstellungen zu Gewerkschaft und Beruf im Verlaufe der verschiedenen Lebensphasen verändern, von der Single-Phase (mit erhöhter Flexibilitätsfähigkeit) über die Familienphase (mit erhöhten Sicherheitsansprüchen) bis zum erhöhten Ausgliederungsrisiko der mehr als Fünfzigjährigen (mit erhöhten Schutzansprüchen).

Für mögliche Gegenstrategien markieren das neue Entgeltrahmenabkommen
(ERA) wie das Pforzheimer Abkommen der IG Metall eine Wende. Bei Beibehaltung des Flächentarifvertrags erlauben sie eine flexiblere und dezentrale Berücksichtigung der betrieblichen Bedingungen, der Qualifikationsunterschiede der verschiedenen Beschäftigtengruppen, der Weiterbildung und der Mitbestimmung. Dies ist zugleich eine Chance und ein Problem. Bei dem Weg zu einer "nachfordistischen“ Tarifstrategie handelt es sich um einen Suchprozess. Das schließt gelingende und auch misslingende Versuche ein, den verschiedenen Teilgruppen einer immer differenzierteren Arbeitnehmerschaft gerecht zu werden.

\section{Untersuchungsmethode: Exploration eines sich wandelnden Feldes}

Die Pilotstudie sollte die entsprechenden Veränderungen im typologischen Feld höher qualifizierter Arbeitnehmermilieus explorieren. Die Grundstruktur des typologischen Gesamtfelds der sozialen Milieus ist bekannt. Hier ist sie anhand einer „Milieulandkarte" (Abbildung 1) vereinfacht abgebildet. Die Milieutypen sind in den 1990er Jahren aus repräsentativen Daten entwickelt und dann im sozialen Raum angeordnet worden (Bourdieu 1982; Vester et al. 2001; Vögele et al. 2002).

Abbildung 1 zeigt diese Milieukonstellation, hochgerechnet auf die Größen von 2003. Jedes Milieu hat Schwerpunkte in bestimmten Berufsfeldern. So dominieren in den oberen Milieus Akademiker und Führungskräfte, in der großen Arbeitnehmermitte die qualifizierten Arbeiter und Angestellten und bei den unterprivilegierten Milieus die an- und ungelernten Tätigkeiten.

Horizontal bildet sich von rechts nach links die Entwicklung zu moderneren Berufen $\mathrm{ab}$, die mehr Eigenverantwortung und Berufsqualifikation erfordern. Dadurch haben sich die modernen Arbeitnehmermilieus, die erst seit den 1980er Jahren entstanden sind, als einzige und sehr rasch vergrößert - auf zusammen $23 \%$ der Bevölkerung. Von diesen hat das „Moderne Arbeitnehmermilieu" den Schwerpunkt in technischen, organisatorischen und sozialen Schrittmacherberufen und das „Moderne kleinbürgerliche Arbeitnehmermilieu" den Schwerpunkt in moderneren kaufmännischen und verwaltenden Berufen. In ihrem Umkreis hat das „Bildungskapital“ besonders zugenommen. (Die „Landkarte" verzeichnet, oval umrandet, auch die Abitur- und Hochschulabschlüsse der verschiedenen Teilmilieus.)

Die neuen Struktur- und Statusverschiebungen, Differenzierungen und Konfliktlinien, die dieses Bild inzwischen verändern, sind jedoch noch nicht hinreichend untersucht. Die Pilotstudie sollte daher die Veränderungen dieser moderneren, hoch qualifizierten Arbeitnehmermilieus und ihrer Nachbarmilieus neu explorieren (und zu Vergleichszwecken auch das klassische Facharbeitermilieu einbeziehen). 
Untersucht werden sollten die neuen Konfliktkonstellationen nach dem Konzept von Habitus und Feld von Pierre Bourdieu (1982; 1987) und der daran anknüpfenden typenbildenden Mentalitäts- und Milieuanalyse (Vester et al. 2001; Bremer 2004). Dabei geht es um die Spannung zwischen den inneren Handlungsdispositionen einer Akteursgruppe (dem Habitus- oder Mentalitätstypus, zentriert im Berufsethos) und den äußeren Handlungszumutungen (den gewandelten Arbeits- und Lebensverhältnissen, zentriert in der Betriebssituation). Mit explorativen, d.h. ,strukturentdeckenden "Verfahren können diese konfliktgenerierenden Spannungen zwischen beiden Seiten sichtbar gemacht werden, auch in Dimensionen, die bisher weniger bekannt und erkennbar sind. ${ }^{5}$

Die mit der neuen Statusunsicherheit verbundenen Konflikte sind mit dem Konzept des Habitus vollständiger zu erfassen als mit Konzepten der rein materiellen Lebens- und Sicherheitsstandards. Beim Status eines sozialen Milieus geht es jeweils um die Sicherung einer ganzen Lebensweise, zu der klar umrissene Präferenzen des Geschmacks, des Berufsethos, der Lebensführung, der Bildungs- und Berufsstrategien gehören. Und ebenso geht es um Vorstellungen über den Platz des eigenen und der anderen Milieus in der sozialen Ordnung als Ganzes. Der Habitus fasst diese Dimensionen der sozialen Identität ganzheitlich zusammen.

Der Kern der neuen Konfliktkonstellationen liegt - so unsere Hypothese - darin, dass durch den Strukturwandel des Wirtschaftssystems viele Milieus ihren bisherigen gewohnten Status infrage gestellt sehen. Sie können sich darauf nur begrenzt umstellen, da ihr Habitus in der Regel auf eine festgelegte, in den Familien über lange Zeiten weitergegebene Weise auf einen bestimmten sozialen Status abgestimmt ist. Diese Passungen zwischen Habitus und Feld sind durch den neuen Strukturwandel ins Rutschen gekommen.

Der Strukturwandel sollte an einem ausgewählten typologischen Spektrum exportorientierter Elektronik-, Maschinenbau- und Automobilunternehmen und den hierfür relevanten Ausbildungseinrichtungen untersucht werden. Dabei wurden Unternehmen berücksichtigt, die die verschiedenen unternehmerischen Strategien der Umstellung auf den internationalen Wettbewerb prototypisch abbilden. Entsprechend der Fragestellung wurde die Auswahl

\begin{tabular}{|ll|}
\hline Übersicht 1: Die Erhebungsgruppen & \\
\hline Erhebungsgruppe & Erhebungsort/ Branche \\
\hline Systemelektroniker (Ausbildung) & Berufsbildende Schule \\
Fachinformatiker (Ausbildung) & Berufsbildende Schule \\
Studierende Ingenieurwissenschaften & Technische Universität \\
Ingenieure - Berufseinsteiger & Automobilindustrie \\
Ingenieure 35 plus & Elektronik-Branche \\
Qualifizierte Facharbeiter(innen) & Maschinenbau \\
Kaufmännische Angestellte mittlerer Qualifikation & Fahrzeugbau (Baumaschinen, Nutzfahrzeuge) \\
\hline Quelle: Angaben der Autoren. & \\
\hline
\end{tabular}

der Betriebe kombiniert mit der Auswahl der Berufsmilieus: Ingenieure und Informatiker, qualifizierte Facharbeiter und kaufmännische Angestellte. Ebenso wurden verschiedene Berufsphasen berücksichtigt: von Ausbildung und Studium über Berufseinsteiger bis in die höheren Altersgruppen (Übersicht 1).

\section{Untersuchungsergebnisse}

Aus den Erhebungen konnten fünf Arbeitnehmertypen mit ausführlichen Typenporträts, zentriert um das Berufsethos, herausgearbeitet und in den sozialen Raum eingeordnet werden (Abbildung 2). Die begrenzte Stichprobe der Pilotstudie erlaubt lediglich erste Einblicke, verdeutlicht aber doch exemplarische Konfliktlinien. Im Folgenden gehen wir auf sechs Punkte näher ein.

\subsection{STRATEGIEN: INTEGRATION ODER REGLEMENTIERUNG}

Die untersuchten Unternehmen stellen sich dem globalen Wettbewerb mit unterschiedlichen Strategien, die teils im Einklang und teils im Konflikt mit dem Berufsethos der Befragten stehen.

Einige Unternehmen mobilisieren eigenverantwortliche Kompetenzen durch integrative Strategien, die den Gegensatz zwischen Management und Beschäftigten durch akzeptanzfähige Aushandlungen und Regelungen moderieren. Dies sind einerseits kleinere IT-Unternehmen mit flachen Hierarchien, die die Mitarbeiter fast partnerschaftlich in Entscheidungsprozesse einbeziehen, andererseits ein Maschinenbau-Unternehmen mit einem paternalistischen Modell der „Betriebsfamilie“. (Eine andere integrative Strategie ist der von Schumann et al. (2006) in der Studie „VW Auto 5000“ analysierte Produktivitäts- und
Mitbestimmungspakt, der mit der Interessenvertretung ausgehandelt ist.)

Verschiedene große Unternehmen bevorzugen dagegen von oben durchgesetzte betriebswirtschaftliche Strategien der Reglementierung. Sie fördern primär nicht eine höhere Produktivität durch intelligentere Arbeitsprozesse, sondern eine billigere Produktion, durchgesetzt über einen steigenden Kosten-, Zeit- und Flexibilitätsdruck. Diese Politik wurde teilweise bis zum Punkt der Kontraproduktivität gesteigert und hat ein erhebliches Konfliktpotenzial bei den technischen Experten erzeugt. Als Überreglementierung demobilisiert sie ihr produktives Potenzial. Dies trifft insbesondere auf den Typus „Gebremste Technische Experten " $\mathrm{zu}$ - Ingenieure und Techniker in den Forschungs- und Entwicklungszentren der Automobil- und Elektronikbranche. Das von den hoch Qualifizierten geforderte unternehmerische Denken und Handeln wird durch ein System undurchsichtiger Zielvereinbarungen, Vorgaben, Kontrollen und Bürokratismen untergraben. Ein jüngerer Ingenieur: „Es gibt immer mehr Bereiche um mich rum, wo ich das Gefühl habe, die arbeiten nicht, aber die verfolgen mich."

\subsection{KONFLIKTE: „TECHNISCHE ARBEITSLOGIK" VERSUS "BETRIEBSWIRTSCHAFTLICHE ORGANISATIONSLOGIK"}

An diesem Typus zeigt sich ein verschärfter grundsätzlicher Konflikt, der auch für an-

\footnotetext{
5 Die Erhebungen folgten der Methode der mehrstufigen Gruppenwerkstatt, mit der die beruflichen Ansprüche und Anforderungen, Zukunfts vorstellungen sowie Erfahrungen und Wahrnehmungen hinsichtlich der Unternehmensführung und der Gewerkschaften erhoben und über assoziativ-projektive Techniken, wie die Collagentechnik, auch tiefer liegende und weniger reflektierte Ebenen von Habitus und Berufsethos exploriert wurden (Bremer/Teiwes-Kügler 2003; Bremer 2004). Die Auswertung erfolgte nach der Methodik der typenbildenden Habitushermeneutik.
} 


\section{Abb. 2: Moderne Arbeitnehmer im sozialen Raum Westdeutschlands 2005}

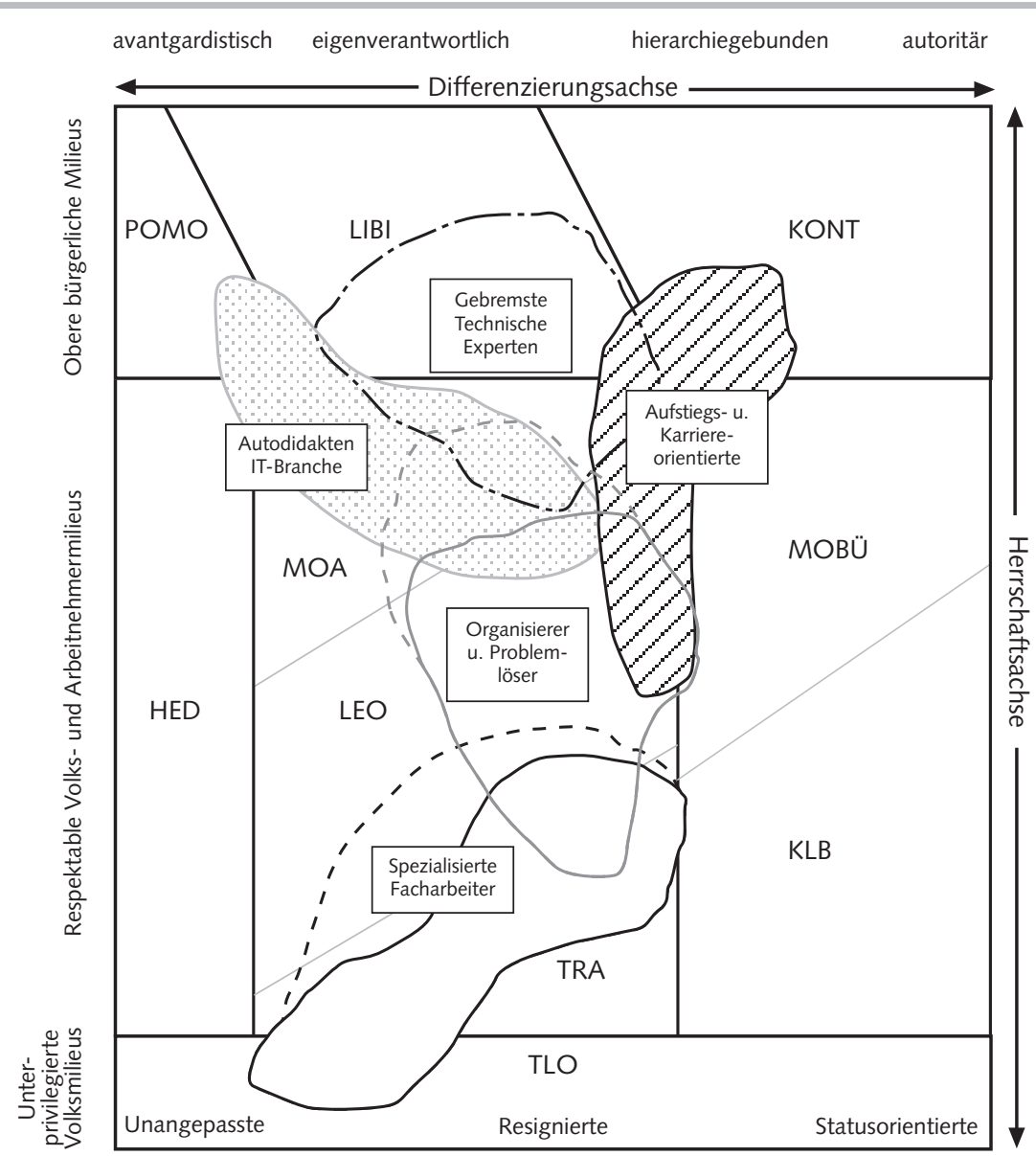

LIBI: Liberal-intellektuelles Milieu (ca $8 \%$ )

KONT: Konservativ-technokratisches Milieu (ca. 7 \%)

POMO: Postmodernes Milieu (ca. $6 \%$ )

HED: Hedonistisches Milieu (ca. $9 \%$ )

MOBÜ: Modernes, kleinbürgerliches Arbeitnehmermilieu (ca. $12 \%$ )

Die gestrichelten Linien in der Abbildung (- - - ) geben die hypothetische Ausdehnung der Typen an, die durch die Stichprobe der Pilotstudie bislang noch nicht abgedeckt wurden.

Quelle: Eigene Darstellung der Autoren.

WSI MITTELLUNGEN

dere technische Fachkräfte zutrifft. Gemäß ihrem ausgeprägten Berufsethos folgen sie bei ihrer Entwicklungstätigkeit einer auf langfristige Erfahrungskumulation ausgerichteten „technischen Arbeitslogik“ (Oesch 2006), die Zeit und Raum für Eigenverantwortlichkeit, Kreativität und experimentelles Vorgehen lässt und sich an der Qualität der Produkte und an fachlichem Expertenwissen orientiert. Immer häufiger gerät dieses Berufsethos in Widerspruch zur betriebswirtschaftlichen „Organisationslogik“ (Oesch 2006), die auf kurzfristige Kostenvorteile des „shareholder value" zielt. Hinter diesen Profitmaximierungsstrategien müssen gegebenenfalls Qualitätsansprüche zurückstehen. Ein Ingenieur: „Da kam dieser Herr und hat ge- wirtschaftlichen Orientierungen. Dieser „aufstiegs- und karriereorientierte“ Typus strebt Führungspositionen an, die mit Prestige, Status und Macht verbunden sind: „Das technische Interesse stand nicht so im Vordergrund, sonst hätte ich ja Ingenieurwissenschaften studiert (...). Ich wollte halt was machen, wo man dann später auch mal was entscheiden kann und nicht nur der Zuarbeiter ist" (Interview).

Die besondere Produktivkraft der „Technischen Experten“ ist ihr über lange Zeit erworbenes informelles Erfahrungswissen. Dieses findet immer weniger Anerkennung, was Ausdruck der aktuellen Positionskämpfe mit aufsteigenden und etablierten Managementfraktionen ist. $\mathrm{Zu}$ nehmend werden die „Technischen Experten", die häufig Bildungsaufsteiger sind, abgedrängt: „Früher hatte man zwei mögliche Wege der Karriere. Ein Weg war der Weg als Fachspezialist, wo man in seinem Gebiet anerkannt wurde, da konnte man nach oben steigen. Diesen Weg gibt's nicht mehr, anerkannt ist nur noch der Erfolg an Umsatz, an Menschenführung und so weiter. Sklavenhändler, die können damit gut umgehen" (Interview).

Insbesondere ältere Ingenieure fürchten Arbeitsplatzverlust und Deklassierung, da die Elektronik- und Hightech-Firmen ihre Forschungs- und Entwicklungszentren zunehmend ins Ausland verlegen. Die Arbeitslosenzahlen bestätigen das erhöhte Risiko für ältere Ingenieure. Verunsicherungen und Entwertungserfahrungen sind bei den hoch Qualifizierten angekommen. Damit hat sich der Arbeitnehmer-Arbeitgeber-Gegensatz verstärkt. In einer Collage offenbarte die Gruppe diese Ängste mit dem Foto eines plötzlich auf der Landstraße auftauchenden Hirschs und dem Satz: „Rechnen Sie mit allem“. Ein Ingenieur dazu: „Ich will nicht mit allem rechnen, wenn ich älter werde. Ich will schon irgendwie eine gewisse Sicherheit haben, dass ich mein Haus nicht verkaufen muss oder egal was, wenn ich da auf der Straße liege ..., dass man zu etwas gezwungen wird, wo man in Verzweiflung reinkommt" (Interview).

Die Entwertung des Erfahrungswissens beschäftigt auch die „Autodidakten der ITBranche", junge Fachinformatiker in der Berufsausbildung. Sie waren vorher häufig auf der Grundlage autodidaktisch erworbener Kompetenzen als „Pioniere“ in der Branche tätig und müssen nun eine formelle Ausbildung absolvieren, um sich weiter beruflich positionieren zu können. Der 
Hauptkonflikt besteht für sie nicht im Interessengegensatz zu den Unternehmen. Hier konnten Ansprüche auf Individualität und eigenverantwortliche Mitgestaltung realisiert werden. Der Hauptkonflikt besteht eher in der Umbruchsituation der Branche selbst. Im Zuge der Professionalisierungsund Verstetigungsprozesse fordert diese zunehmend qualifizierte Ausbildungen, zertifiziertes Wissen und auch akademische Berufsabschlüsse.

Dem entspricht ein Dauerkonflikt mit den Lehrkräften der berufsbildenden Schulen, gekennzeichnet durch mangelnde gegenseitige Anerkennung. Dies ist Ausdruck symbolischer Kämpfe zwischen selbstbewussten praktischen Milieus der Arbeitnehmermitte und Teilen der privilegierten akademischen Milieus. Semiprofessionelle Berufsgruppen wie die Fachinformatiker werden auf „Ersatzkarrieren“ verwiesen, die auch bei permanenter Weiterqualifizierung relativ ungesichert bleiben. Ohne akademischen Abschluss sind Positionen mit höheren Einkommen, Privilegien, Ansehen und besserer sozialer Absicherung nicht erreichbar.

Der Typus gibt sich heute selbstbewusst und aufgeschlossen für neue Herausforderungen und Flexibilität. Langfristig wünscht er sich doch, insbesondere für die Familiengründung, eine gesicherte, planbare Existenz. Zum Textmotiv „Anschlusshalten " in der Collage erläutert ein Fachinformatiker: „Man hat den Spaß dabei ..., weil man ja das Interesse an dieser Technologie hat, aber aufder anderen Seite ist es unheimlich schwierig, in einer derart schnell wachsenden Branche ... den Anschluss immer zu kriegen, um nicht $z u$ sagen, hier an dem Punkt bin ich auf der Strecke geblieben. ... Das wird auf jeden Fall richtig anstrengend. “ Zum Textmotiv „Security“ erklärt ein anderer: "Das ist mehr dann so ' $n$ Wunsch, dass ich mir Sicherheit auch wünsche ... etwas, was man so wahrscheinlich nie mehr kriegen wird" (Interview).

\subsection{UNTERNEHMENSKULTUR: DAS ZUSAMMENSPIEL VON UNTERNEHMENSSTRATEGIEN UND BESCHÄFTIGTENSTRUKTUR}

Das Management kann die Kultur und Spielregeln des Unternehmens nicht einseitig verändern, sondern nur, wenn dem Strukturen der Produktionsweise und der Belegschaft entgegenkommen. Beispielsweise muss der Typus „Organisierer und
Problemlöser", der mit mittleren, meist technischen Qualifikationen an Gelenkstellen einer spezialisierten Maschinenfabrik eingesetzt ist (Disposition, Beschaffung, Lagerhaltung), eigenständig unter Zeitdruck Probleme managen, damit Abteilungen und Produktion störungsfrei ineinander greifen. Mit einer gemeinschaftsorientierten Strategie, einer Mischung aus permanenter Beziehungspflege, kooperativem Umgangsstil und gelegentlichem Druck, halten die „Organisierer und Problemlöser“ den Betrieb zusammen. Sie können dabei auf eine seit Generationen überlieferte solidarische Facharbeiterkultur zurückgreifen. Das neue, japanische Management hat diese geschickt in das Konzept einer betrieblichen Familie eingebunden.

Inzwischen aber verschiebt sich das Kräfteverhältnis zwischen dem bisher dominanten gemeinschaftlich-solidarischen (technischen) Typus und neu hinzukommenden kaufmännisch-organisatorisch orientierten Beschäftigten. Diese sind besser ausgebildet und qualifiziert und setzen stärker auf Konkurrenz und individuellen Aufstieg. Ihr Habitus hat mehr Nähe zu den inzwischen vom Unternehmen eingeführten individuellen Leistungsanreizen, die Konkurrenzen verstärken. Dieser „neue“ Typus wird vermutlich längerfristig den Einfluss des gemeinschaftlichen Typus auf die Betriebskultur zurückdrängen.

\subsection{INTERESSENVERTRETUNG: KOLLEKTIVE DELEGATION UND INDIVIDUELLE SELBSTVERTRETUNG}

Bei den Berufsanfängern haben die Chancen der Gewerkschaften, neue Mitglieder $\mathrm{zu}$ werben, abgenommen, seitdem sich die außerbetrieblichen Ausbildungsphasen verlängern. Eine frühe betriebliche Sozialisation durch gewerkschaftlich organisierte Kollegen entfällt heute vielfach, etwa bei Auszubildenden der IT-Branche oder Studierenden. Zudem ist höher qualifizierten Berufsanfängern individuelle Flexibilität eher möglich. Das Interesse an einer arbeitnehmerischen Interessenvertretung nimmt aber in der Familienphase wieder $\mathrm{zu}$, wenn berechenbare Arbeits- und Einkommensverhältnisse gebraucht werden.

Bei den technischen Experten und Fachkräften verschiebt sich die gewünschte Interessenvertretung von der kollektiven Delegation zur individuellen Selbstvertretung. Zum Berufsethos gehört, für die eigenen Interessen auch selbst einzustehen.
Die Ergänzung durch solidarische Interessenvertretung wird, wenn sie nötig ist, aber keineswegs abgelehnt. Die höher Qualifizierten geraten mit der individuellen Selbstvertretung gerade in größeren Unternehmen an Grenzen, wenn sich die Konflikte mit dem Management verschärfen. Aber sie haben in manchen Betrieben den Eindruck, dass die Gewerkschaftsvertreter noch nicht hinreichend kompetente Partner sind, um ihre Anliegen wirksam genug wahrnehmen zu können. Auch bestehen häufig noch kulturelle Barrieren gegenüber gewerkschaftlichen Auseinandersetzungsstilen. Die Rituale der Kampfkraft gelten noch als zu konfrontativ, zu wenig am Betriebswohl und integrativen Aushandlungsstilen orientiert. Nicht in allen, aber in vielen Betrieben haben Gewerkschafter und Betriebsräte trotz sichtlichen Bemühens noch keinen Weg gefunden, die technischen Experten und Fachkräfte, die sich auf dem Weg von privilegierten zu normalen unsicheren Arbeitnehmerlagen befinden, hinreichend zu unterstützen.

\subsection{ENTFREMDUNG: DIE GEWERKSCHAFTEN UND IHRE KERNKLIENTEL}

Entfremdungen sind heute ebenfalls zwischen den Gewerkschaften und ihrer alten Kernklientel möglich, vor allem den klassischen „Spezialisierten Facharbeitern“, mit ihrem ausgeprägten Fachethos und Klassenbewusstsein, das die Interessengegensätze zwischen Arbeit und Kapital und auch zwischen Angestellten und Arbeitern betont. Die befragten Facharbeiter betrachten sich immer noch als diejenigen, die mit ihrer sichtbaren produktiven Arbeit die eigentliche Wertschöpfung erbringen und den „Wasserkopf " der Angestellten mittragen.

Doch ihr auf handwerkliches Fachkönnen gestütztes Selbstbewusstsein gerät durch die Zunahme der Angestellten und die fortschreitende Verlagerung produzierender Bereiche ins Ausland ins Wanken. Sie nehmen dies als Privilegierung des Angestelltensektors und als Entwertung der qualifizierten Facharbeit wahr: „Heute gibt es nur noch Dienstleistung. Die alte Facharbeit läuft aus" (Interview). Sie haben sich einen bescheidenen Wohlstand erarbeitet, sind gut informiert (sie lesen regelmäßig lokale Tageszeitung, Spiegel, Fokus und GEO) und analysieren im Rahmen ihres autodidaktischen Arbeiter- und Handwer- 
kerintellektualismus (Weber 1972) die Verhältnisse. Nun fürchten sie, durch den Strukturwandel ihre Respektabilität zu verlieren und in die unterprivilegierten Milieus abzurutschen. Da sich die Gewerkschaften aber dem wachsenden Angestelltenbereich verstärkt zuwenden müssen, ist es bedeutsam, auf welche Weise die Moderation, Arbeitsbewertung und Konfliktregulierung erfolgt.

Im Zusammenhang mit dem Entgeltrahmenabkommen ERA haben in dem untersuchten Betrieb, wie auch anderswo, die Unternehmer versucht, Abgruppierungen qualifizierter und höher qualifizierter Arbeitskräfte auf das Niveau einfacher bzw. standardisierter Arbeit durchzusetzen. Auch im Zusammenhang mit dem „Pforzheimer Abkommen", das bedingte Abweichungen vom Flächentarifvertrag ermöglicht, kam es zu Konflikten. Häufig versuchen Arbeitgeber, Abweichungen nach unten durchzusetzen, ohne nach dem Tarifvertrag vorgesehene Gegenleistungen (Beschäftigungsgarantien, Investitionen usw.) anzubieten.

Die betrieblichen Interessenvertretungen können dem dort entgegenhalten, wo es ihnen gelingt, sich aktiv auf die Gewichtsverschiebung zur betriebsnahen Tarifpolitik umzustellen. Hier können sie der Unzufriedenheit von Teilgruppen entgegenwirken und auch neue Mitglieder gewinnen, wenn sie sich als Experten einer kompetenten Arbeitsbewertung, Beschäftigungssicherung, Mitgliederbeteiligung und betrieblichen Konfliktstrategie profilieren. Das Gelingen einer betriebsnäheren Tarifpolitik ist ein wesentlicher Faktor dafür, ob sich der Typus der spezialisierten Facharbeiter weiterhin integriert oder als "Auslaufmodell“ an den Rand gedrängt sieht. Sie erwarten, in Entscheidungsprozesse einbezogen zu werden und ihre Stellung als Facharbeitergruppe mit ihren Ansprüchen an hochwertige Facharbeit rechtlich gesichert zu bekommen.

\section{Alternativen: neoliberal, ständisch oder arbeitnehmerisch}

Die Pilotstudie hat steigende Unruhe in allen Schlüsselgruppen arbeitnehmerischer Leistungsträger festgestellt. Dabei geht es nicht um eine „psychologische Befindlich- keit", sondern um reale Statusverluste, nicht nur um „materielle Verteilung“, sondern auch um Mitbestimmung am Arbeitsplatz und Leistungsgerechtigkeit. Diese Ansprüche sind fest im Berufsethos verankert und nicht durch eine bloße Psychologie des „Betriebsklimas“ oder der „Betriebsfamilie“ zufriedenzustellen.

Hinter der neuen Statusunsicherheit stehen tiefe Verschiebungen der Sozialstruktur. Die internationale Konkurrenz beschleunigt den Trend zur „Hochqualifikationsökonomie“. Eine Qualifikationsschere treibt die Arbeitnehmermitte auseinander. Nach unten wird ein neues Prekariat von gering Qualifizierten erzeugt. Nach oben wachsen die höher qualifizierten Arbeitnehmergruppen; die jüngeren Generationen der Facharbeiter und Fachangestellten stellen ihre Ausbildungswege auf den wachsenden Bedarf in Industrie und Dienstleistungen um.

Aber die Träume von Aufstieg, Eigenständigkeit und Sicherheit erfüllen sich damit nicht. Statt einer gehobenen „ständischen" Sicherheit finden sie die normale "arbeitnehmerische" Unsicherheit: Ihre Arbeitskraft ist eine austauschbare Ware wie jede andere. Damit wächst wieder das Bedürfnis nach einer Interessenvertretung. Diese Erfahrung machen sowohl die Arbeitnehmer, die nach oben wollen, als auch die, die schon oben sind.

Die schon oben sind, die älteren akademischen Experten, sind schon früher in der Geschichte der Bundesrepublik aus der Arbeitnehmermitte in das „Gehobene Dienstleistungsmilieu“ (Abbildung 1) aufgestiegen. Sie haben noch die gehobene soziale Sicherheit (auch beim Älterwerden), die Anerkennung ihres Berufsethos und das Vertrauensverhältnis mit der Unternehmensführung verinnerlicht. Diese ständischen Sicherheiten sehen sie nun dort bedroht, wo Firmen auf neoliberale Strategien der Flexibilisierung, Kostensenkung, Arbeitszerlegung, Verkürzung von Entwicklungszeiten und Auslagerung der Arbeitsplätze in andere Länder setzen. Diese Strategien sichern kurzfristige Vorteile, werden aber kontraproduktiv, wenn sie - wie häufig beobachtet - die Voraussetzungen einer Qualitätsproduktion und damit die $\mathrm{Zu}$ friedenheit nicht nur der Arbeitskräfte („Berufsethos“), sondern auch der Kunden („Produktvertrauen“) aufs Spiel setzen.

Die jüngeren technischen Experten im rasch wachsenden „Modernen Arbeitnehmermilieu“ erfahren, dass sie zwar in der flexiblen Single-Phase höhere individuelle Chancen haben, doch in der Familienphase in die Schere zwischen dem raschen technischen Wandel und der Notwendigkeit eines berechenbaren Einkommens geraten werden. Zudem wird der Aufstieg in besser gesicherte akademische Positionen erschwert durch eine zunehmend rigide Stufung der formellen beruflichen Ausbildungsabschlüsse.

Dies alles sind keine vom Markt diktierten Naturprozesse, sondern die Ergebnisse der Gestaltung durch Interessengruppen und Politik. Wenn immer mehr höher qualifizierte Experten gebraucht werden, stehen wir vor einer gesellschaftspolitischen Alternative. Die Erfüllung des Wunsches nach mehr Anerkennung, Gestaltungsspielräumen, Einkommen und Sicherheit würde die gesellschaftliche Integration und die wirtschaftliche Produktivität der hoch qualifizierten Arbeitnehmergruppen steigern. Aber sie würde auch die konservative Hierarchie durch mehr Mitwirkung von unten infrage stellen.

Auch die Konservativen sehen das neoliberale Modell als einen Quell kontraproduktiver sozialer Reibungen und Konflikte. Sie ziehen ein ständisch gestuftes Ordnungsmodell vor, das jedem einen Platz gibt, aber nicht zu viele nach oben gelangen lässt. Die Strategie der Betriebsfamilie, wie beispielsweise bei Toyota, nutzt die produktiven Kompetenzpotenziale der Belegschaft über eine paternalistische Unternehmenskultur, die Sicherheit, aber doch eine steil gestufte Hierarchie und wenig aktive Mitgestaltung bietet. Im Bildungssystem entspricht dem die Einziehung neuer Zwischenetagen der Fachschulung, des Bachelors usw., durch die die obersten Gruppen klein gehalten werden.

Eben hierzu bietet die neue, differenzierende und betriebsnähere gewerkschaftliche Aushandlungs- und Mitbestimmungsstrategie eine Alternative (Schumann et al. 2006). Sie kann nach dem Prinzip „Leistung gegen Teilhabe", das den Kern des historischen Sozialmodells der Bundesrepublik bildete (Vester et al. 2001), eine arbeitnehmerische Gegenmachtpolitik begründen, die Leistung und Weiterbildung als Beiträge zu höherer Produktivität honoriert. Damit würde an die Stelle eines Gewinner-Verlierer-Modells wieder ein Modell treten, in dem beide Seiten gewinnen in gewisser, wenn auch nicht unbegrenzter Analogie zu dem Win-Win-Modell der Wachstumsjahre nach 1945. 
Ohne dass die gering qualifizierten Gruppen unwichtig würden, nimmt die Bedeutung der Arbeitnehmer mit zunehmendem Qualifikationsrang und Autonomieanspruch zu. Sie verlieren ihre ständischen Privilegien und brauchen eine ar- beitnehmerische Interessenvertretung, die ihrem Anspruch an Stil, Kompetenz und Selbstvertretung entgegenkommt. Individuelle und kollektive Interessenvertretung sind kein Gegensatz, wenn die Gewerkschaft differenziert und flexibel vorgeht und diese Politik auf den gemeinsamen Nenner der Qualitätsproduktion bringt wie dies in dem Grundsatz „, besser statt billiger" zum Ausdruck kommt.

\section{LITERATUR}

Baethge, M./Denkinger, J./Kadritzke, U. (1995): Das FührungskräfteDilemma, Frankfurt/M./New York

Beerhorst, J./Berger, J.-J. (Hrsg.) (2003): Die IG Metall auf dem Weg in die Mitte?, Hamburg

Blauner, R. (1964): Alienation and Freedom, Chicago/London

Bourdieu, P. (1982): Die feinen Unterschiede, Frankfurt/M.

Bourdieu, P. (1987): Sozialer Sinn, Frankfurt/M.

Bremer, H. (2004): Von der Gruppendiskussion zur Gruppenwerkstatt, Hamburg

Bremer, H./Teiwes-Kügler, Chr. (2003): Die Gruppenwerkstatt, in: Geiling, H. (Hrsg.): Probleme sozialer Integration, Münster, S.207-236 Durkheim, É. (1988 [1893/1902]): Über soziale Arbeitsteilung, Frankfurt/M.

Durkheim, É. (1999): Physik der Sitten und des Rechts, Frankfurt/M Faust, M./Jauch, P./Notz, P. (2000): Befreit und entwurzelt: Führungskräfte auf dem Weg zum „internen Unternehmer ${ }^{4}$, München/Mering Grusky, D. B/Soerensen, J. B. (2001): Are There Big Social Classes?, in: David B. Grusky (ed.): Social Stratification. $2^{\text {nd }}$ ed. Boulder/Col., pp. 183194

Huber, B. (2003): Gewerkschaftspolitische Schlussfolgerungen aus der Differenzierung der Arbeitnehmerschaft, in: Beerhorst, J./Berger, J.-J. (Hrsg.): Die IG Metall auf dem Weg in die Mitte?, Hamburg, S.128-134 IG Metall (2004): FB Beitrags- und Leistungswesen Metallwirtschaft, Frankfurt/M., Mai

Kern, H./Schumann, M. (1984): Das Ende der Arbeitsteilung?, München Kotthoff, H. (1998): Führungskräfte im Wandel der Firmenkulturen, Berlin
Mallet, S. (1972): Die neue Arbeiterklasse, Neuwied/Berlin Oesch, D. (2006): "Coming to Grips with a Changing Class Structure“, in: International Sociology 2, pp. 263-288

Piore, M. J./Sabel, Ch. F. (1985): Das Ende der Massenproduktion, Berlin Schumann, M./Kuhlmann, M./Sanders, F./Sperling, H. J. (Hrsg.) (2006): Auto 5000: Ein neues Produktionskonzept, Hamburg Sigma - Sozialwissenschaftliches Institut für Gegenwartsfragen (2003): Die sozialen Milieus in der Verbraucheranalyse, www.sigma-online.com, vom 22.9.

Vester, M. (2003): Wie differenziert ist die Arbeitnehmermitte?, in: Beerhorst, J./Berger, J. J. (Hrsg.): Die IG Metall auf dem Weg in die Mitte?, Hamburg, S. 101-124

Vester, M. (2005): Die selektive Bildungsexpansion, in: Berger, P. A./Kahlert, H. (Hrsg.): Institutionalisierte Ungleichheiten, Weinheim/München, S. 39-70

Vester, M. (2006): Die gefesselte Wissensgesellschaft, in: Bittlingmayer, U. H./Bauer, U. (Hrsg.): Die Wissensgesellschaft, Wiesbaden Vester, M./v. Oertzen, P./Geiling, H./Hermann, Th./Müller, D. (2001): Soziale Milieus im gesellschaftlichen Strukturwandel, Frankfurt/M. Vögele, W./Bremer, H./Vester, M. (Hrsg.) (2002): Soziale Milieus und Kirche, Würzburg

Weber, M. (1972 [1921]): Wirtschaft und Gesellschaft, Tübingen Weber, M. (1991): Die protestantische Ethik, Hg. v. J. Winckelmann, 8. Aufl., Gütersloh

Weber-Menges, S. (2004): Arbeiterklasse oder Arbeitnehmer?, Wiesbaden 